This item was submitted to Loughborough's Research Repository by the author.

Items in Figshare are protected by copyright, with all rights reserved, unless otherwise indicated.

\title{
Huber's theorem for hyperbolic orbisurfaces
}

PLEASE CITE THE PUBLISHED VERSION

http://dx.doi.org/10.4153/CMB-2009-008-0

PUBLISHER

(c) Canadian Mathematical Society

VERSION

SMUR (Submitted Manuscript Under Review)

LICENCE

CC BY-NC-ND 4.0

REPOSITORY RECORD

Dryden, Emily B., and Alexander Strohmaier. 2019. "Huber's Theorem for Hyperbolic Orbisurfaces". figshare. https://hdl.handle.net/2134/12515. 
This item was submitted to Loughborough's Institutional Repository (https://dspace.lboro.ac.uk/) by the author and is made available under the following Creative Commons Licence conditions.

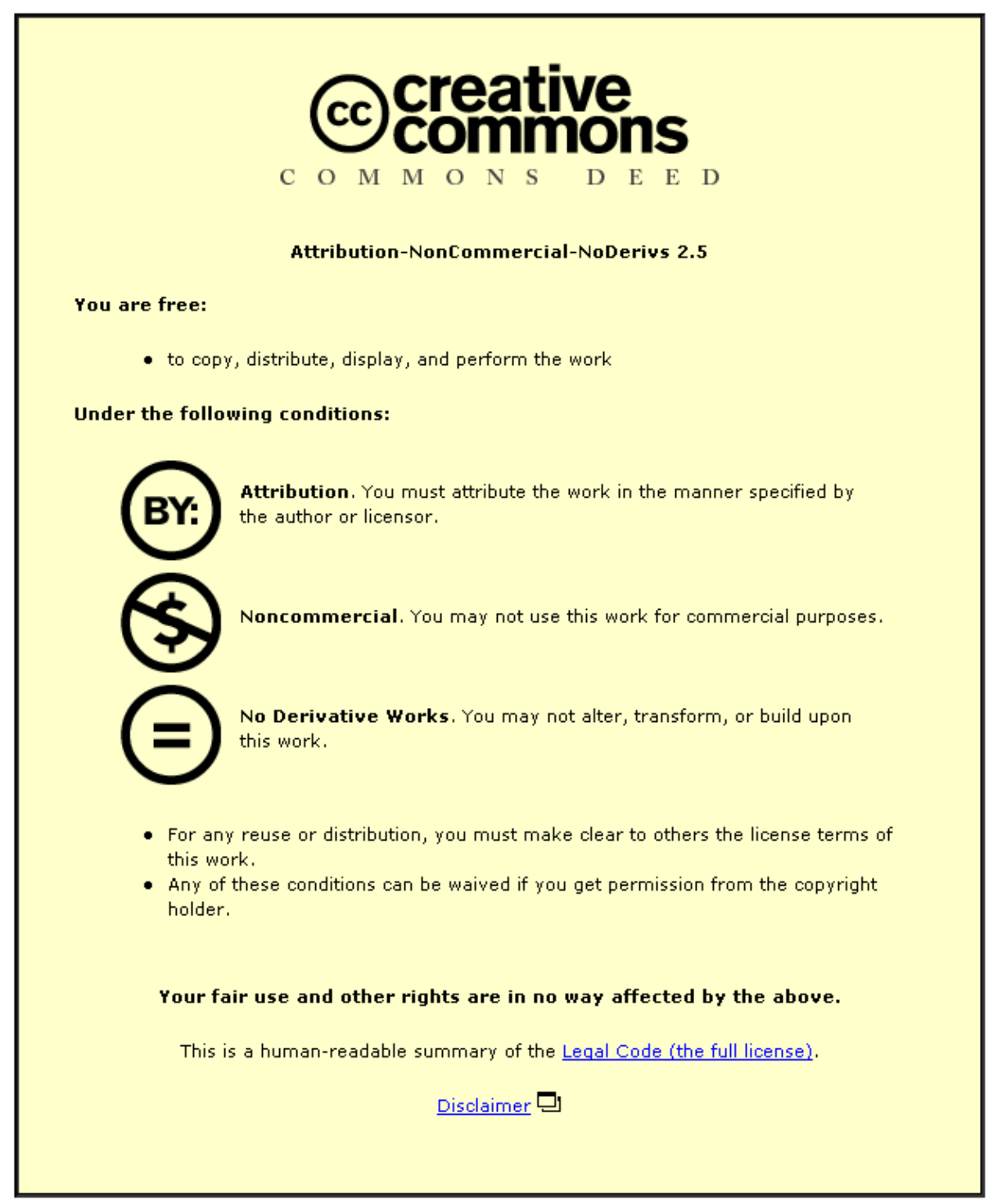

For the full text of this licence, please go to: http://creativecommons.org/licenses/by-nc-nd/2.5/ 


\title{
HUBER'S THEOREM FOR HYPERBOLIC ORBISURFACES
}

\author{
EMILY B. DRYDEN AND ALEXANDER STROHMAIER
}

\begin{abstract}
We show that for compact orientable hyperbolic orbisurfaces, the Laplace spectrum determines the length spectrum as well as the number of singular points of a given order. The converse also holds, giving a full generalization of Huber's theorem to the setting of compact orientable hyperbolic orbisurfaces.
\end{abstract}

Mathematics Subject Classification (2000): 58J53, 11F72

Keywords: Huber's theorem, isospectral, orbisurfaces

\section{Introduction And Result}

We will be interested in compact hyperbolic orbisurfaces, which are a natural generalization of compact hyperbolic Riemann surfaces. By "hyperbolic," we will mean that the object is endowed with a Riemannian metric of constant curvature -1. A hyperbolic orbisurface can be viewed as a quotient of the hyperbolic plane by a discrete group of isometries which is permitted to include elliptic elements. These elliptic elements give rise to conical singularities in the quotient surface. A local neighborhood of a conical singularity looks like the quotient of a disc by the group generated by the rotation through angle $\frac{2 \pi}{n}$ about the disc's center. We call such a singularity a cone point of order $n$, and say that $\mathbb{Z}_{n}$ is the isotropy group associated to the singularity. For background on orbifolds and the eigenvalue spectrum of the Laplace operator in the orbifold context, see [1] and the references therein.

Orbifolds which have the same spectrum of the Laplace operator acting on smooth functions are said to be isospectral. It is known that in general, there can be at most finitely many isotropy groups (up to isomorphism) in a set of isospectral Riemannian orbifolds that share a uniform lower bound on Ricci curvature (see [10]). In fact, N. Shams, E. Stanhope and D. Webb have shown in [9] that there exist arbitrarily large (but always finite) isospectral sets which satisfy this curvature condition, where each element in a given set has points of distinct isotropy. On the other hand, in [2] a spectral invariant is exhibited which, within the class of all teardrops and footballs, determines the number and order(s) of the cone point(s). Teardrops and footballs are orbisurfaces which are topologically the standard sphere $S^{2}$; the teardrop has one cone point, while the football has a cone point at each of the north and south poles, possibly of different orders. These results lead us to ask whether the spectrum determines the orders of the singular points for large classes of orbifolds; we answer this question for the class of compact orientable hyperbolic orbisurfaces.

For compact hyperbolic Riemann surfaces, Huber's theorem says that the Laplace spectrum determines the length spectrum and vice versa, where the length spectrum is the sequence of lengths of all oriented closed geodesics in the surface, arranged in 
ascending order. There has been recent interest in extending Huber's theorem for Riemann surfaces to more general settings. In [3], J. Elstrodt, F. Grunewald and J. Mennicke prove a version of Huber's theorem for discrete cocompact subgroups of $\operatorname{PSL}(2, \mathbb{C})$, while L. Parnovskii [8] states an analog for discrete cocompact subgroups of $\mathrm{SO}_{+}(1, n)$. In both cases, the subgroups are permitted to contain elliptic elements, but it is not shown that the spectrum determines these elements explicitly. We prove that the Laplace spectrum of a hyperbolic orbisurface determines its length spectrum as well as the number and orders of the singular points. Our definition of the length spectrum in this case is as follows: if $X$ is a hyperbolic orbisurface then there exists a discrete subgroup $\Gamma \subset P S L(2, \mathbb{R})$, such that $X$ is isometric to $\Gamma \backslash \mathbb{H}$, where $\mathbb{H}$ the Poincare disk. The unit tangent bundle $T_{1} X$ can be defined as the quotient $\Gamma \backslash P S L(2, \mathbb{R})$. The geodesic flow on $T_{1} X$ can be defined as the right action of the one parameter group

$$
a_{t}=\left(\begin{array}{cc}
e^{t / 2} & 0 \\
0 & e^{-t / 2}
\end{array}\right)
$$

An oriented periodic geodesics of length $l$ is by definition a curve $\gamma: \mathbb{R} \rightarrow T_{1} X$ such that $\gamma(t+l)=\gamma(t)$ and such that $\gamma\left(t_{0}\right) a_{t}=\gamma\left(t_{0}+t\right)$. Curves $\gamma_{1}$ and $\gamma_{2}$ are identified if there is a $t_{0} \in \mathbb{R}$ such that $\gamma_{1}(t)=\gamma_{2}\left(t+t_{0}\right)$. The image of such an orbit under the projection $T_{1} X \rightarrow X=\Gamma \subset P S L(2, \mathbb{R}) / P S O(2)$ is a closed curve in $X$ which is parametrized by arc-length and which is locally length minimizing away from cone points. They may pass through cone points, however. The "length spectrum" is the set of lengths of all periodic geodesics in the orbisurface counting multiplicities.

Theorem 1.1. Let $O$ be a compact orientable hyperbolic orbisurface. The Laplace spectrum of $O$ determines its length spectrum and the number of cone points of each possible order. Knowledge of the length spectrum and the number of cone points of each order determines the Laplace spectrum.

Shortly after preparing this manuscript, we learned that P. Doyle and J. P. Rossetti had proven this result independently.

This work began at the conference "Recent developments in spectral geometry" in Blossin, Germany, in November 2004, and we would like to thank the organizers for their invitation and Andreas Juhl for interesting discussions.

\section{The Proof}

The second statement can be proved as for Riemann surfaces (see [1]). Our proof of the first statement is based on the Selberg trace formula for the wave kernel, that is, the distribution $\operatorname{Tr} \cos \left(t \sqrt{\Delta-\frac{1}{4}}\right)$, where $\Delta$ is the Laplace operator on $O$. Let $\left\{\lambda_{n}^{2}\right\}$ be the sequence of eigenvalues of $\Delta$ and denote as usual $r_{n}^{2}:=\lambda_{n}^{2}-\frac{1}{4}$. Then 
Selberg's trace formula for orbisurfaces (see [6], [7]) reads

$$
\begin{aligned}
\sum_{n=0}^{\infty} h\left(r_{n}\right)= & \frac{\mu(F)}{4 \pi} \int_{-\infty}^{\infty} r h(r) \tanh (\pi r) d r \\
& +\sum_{\substack{\{P\} \\
\text { hyperbolic }}} \frac{\ln N\left(P_{c}\right)}{N(P)^{1 / 2}-N(P)^{-1 / 2}} g[\ln N(P)] \\
& +\sum_{\substack{\{R\} \\
\text { elliptic }}} \frac{1}{2 m(R) \sin \theta(R)} \int_{-\infty}^{\infty} \frac{e^{-2 \theta(R) r}}{1+e^{-2 \pi r}} h(r) d r,
\end{aligned}
$$

where $h$ is any entire function of uniform exponential type and $h(r)=h(-r)$. The sums are over the conjugacy classes of hyperbolic and elliptic elements in $\Gamma$. The norm of the hyperbolic conjugacy class $P$ does not depend on the representative chosen, and is denoted by $N(P)$. We let $P_{c}$ denote the unique primitive hyperbolic conjugacy class such that $P=P_{c}^{l}$ with $l \in \mathbb{N}$. The function $g$ is the Fourier transform of $h$ and thus is a compactly supported smooth function. If $R$ is an elliptic conjugacy class, there exists a unique primitive elliptic conjugacy class $R_{c}$ such that $R=R_{c}^{l}$. The integer $m(R)$ denotes the order of $R_{c}$ and $\theta(R)=\frac{\pi l}{m(R)}$ where $1 \leq l \leq m(R)-1$. We may identify the set of primitive elliptic conjugacy classes $R$ in $\Gamma$ with the set of cone points in $O$ of order $m(R)$. The set of hyperbolic conjugacy classes $P$ may be identified with the set of closed periodic geodesics in $O$ of length $\ln N(P)$. Since in the literature this is usually proved for groups without elliptic elements we include the argument here. Let $\gamma(t)=X a_{t}$ be a periodic geodesic with period $T$, where $X$ is in $\Gamma \backslash P S L(2, \mathbb{R})$. Choose a representative $x \in P S L(2, \mathbb{R})$, i.e. $X=[x]$. Since $\gamma$ is periodic with period $T$ there exists a unique element $\gamma \in \Gamma$ such that $x a_{T}=\gamma x$. Since this element is conjugate to $a_{T}$ in $\operatorname{PSL}(2, \mathbb{R})$ it is hyperbolic. It is now easy to see that another representative $x^{\prime} \in P S L(2, \mathbb{R})$ gives rise to a conjugate element $\gamma^{\prime}$ with norm $e^{l}$. Hence, we have a well defined map from periodic geodesics to conjugacy classes of hyperbolic elements. Conversely, if $\gamma \in \Gamma$ is a hyperbolic element, then there is an element $x$ in $P S L(2, \mathbb{R})$ such that $x^{-1} \gamma x=a_{\ln N(P)}$. Hence, $\gamma(t)=[x] a_{t}$ is a periodic geodesic with period $\ln N(P)$. It is easy to see that this geodesic does not depend on the choice of $x$ and on the representative of $\gamma$ in a conjugacy class. This defines thus a map from conjugacy classes of hyperbolic elements in $\Gamma$ to periodic geodesics. This map is clearly the inverse of the above map.

The Selberg trace formula in the form (1) allows us to give a meaning to the wave trace $\Phi(t)=\operatorname{Tr} \cos \left(t \sqrt{\Delta-\frac{1}{4}}\right)$ in the distributional sense. We may define the functional $\Phi$ on $C_{0}^{\infty}(\mathbb{R})$ by

$$
\Phi(f):=\frac{1}{2} \sum_{n=0}^{\infty}\left(\hat{f}\left(r_{n}\right)+\hat{f}\left(-r_{n}\right)\right)
$$

where $\hat{f}$ denotes the Fourier transform of $f ; \hat{f}$ is known to be entire and of uniform exponential type. This defines a distribution in $\mathcal{D}^{\prime}(\mathbb{R})$. Using $(1)$ and the above 
identifications we obtain

$$
\begin{aligned}
\operatorname{Tr} \cos \left(t \sqrt{\Delta-\frac{1}{4}}\right)= & -\frac{\mu(F)}{8 \pi} \frac{\cosh (t / 2)}{\sinh ^{2}(t / 2)}+\sum_{k=1}^{\infty} \sum_{c \in \mathcal{P}} \frac{l_{c}}{4 \sinh \left(k l_{c} / 2\right)}\left(\delta\left(|t|-k l_{c}\right)\right) \\
& +\sum_{x \in \mathcal{C}} \Psi_{m(x)}(t) .
\end{aligned}
$$

Here the first term has to be understood as the distributional derivative of the distribution $\frac{\mu(F)}{4 \pi} \frac{1}{\sinh (t / 2)}$ defined as a principal value (see [5]).

The second term is obtained as follows. $\mathcal{P}$ is the set of oriented primitive closed periodic geodesics; if we took $\mathcal{P}$ to be the set of unoriented primitive closed periodic geodesics, then we would need to sum over $k \in \mathbb{Z} \backslash 0$. So we we changed from a sum over hyperbolic conjugacy classes to a sum over all the iterates of the oriented primitive closed geodesics. We let $l_{c}$ denote the length of $c \in \mathcal{P}$, and we use the fact that

$$
\frac{1}{\sinh \left(\frac{\ln N(P)}{2}\right)}=\frac{2}{N(P)^{1 / 2}-N(P)^{-1 / 2}} .
$$

Finally, the delta function appears when we calculate $g[\ln N(P)]$. In fact, a direct calculation of $g$ yields

$$
g[\ln N(P)]=\frac{1}{2}[\delta(\ln N(P)-t)+\delta(\ln N(P)+t)],
$$

but it is not hard to see that $\delta(|t|-\ln N(P))$ contains the same information as the sum of the two delta functions above.

In the third summand, the set $\mathcal{C}$ is the set of cone points (which correspond to primitive elliptic conjugacy classes), and $m(x)$ denotes the order of $x \in \mathcal{C}$. The functions $\Psi_{m}(t)$ are defined as the Fourier transforms of the functions

$$
\psi_{m}(r):=\sum_{l=1}^{m-1} \frac{1}{4 m \sin (\pi l / m)}\left(\frac{e^{-2 \pi r l / m}}{1+e^{-2 \pi r}}+\frac{e^{2 \pi r l / m}}{1+e^{2 \pi r}}\right) .
$$

Since $\psi_{m}(r)$ is exponentially decaying, the functions $\Psi_{m}(t)$ are real analytic and therefore do not contribute singularities to the wave trace. Knowing the wave trace we can read off the lengths of all primitive closed geodesics from its singular support. This can be done as follows. The first term in the wave trace formula is completely determined by the singularity at $t=0$ (which reflects the fact that the volume is spectrally determined). We subtract it from the right-hand side of (2). Now take the minimal distance, $d$, from 0 to the singular support of the remaining function; $d$ is the length of a primitive closed geodesic, which is contributing to the singularity at $t=d$. From the corresponding wave trace invariant we get the number of primitive closed geodesics contributing to this singularity. Subtract the contribution of those primitive closed geodesics from (2), and again take the minimal distance from 0 to the singular support of the remaining function. Proceed in this way to find all lengths and multiplicities of primitive closed geodesics.

We now know that the spectrum determines the finite sum

$$
\sum_{x \in \mathcal{C}} \Psi_{m(x)}(t)
$$

The functions $\psi_{m}(r)$ behave asymptotically like $\left(2 m \sin \frac{\pi}{m}\right)^{-1} e^{-\frac{2 \pi r}{m}}$ as $r \rightarrow \infty$; from this, we conclude that the $\psi_{m}(r)$ are linearly independent for different values of $m$. 
This in turn implies that the terms contributing to the sum (3) by cone points of different orders are linearly independent. Thus we can read off the orders of the cone points in $O$; the multiplicity of a summand corresponding to a particular order tells us the number of cone points of that order.

\section{Consequences}

Theorem 1.1 has implications for the topology of isospectral hyperbolic orbisurfaces. To describe these consequences, we first need to define the Euler characteristic and state a Gauss-Bonnet theorem for orbisurfaces (see [11]).

Definition 3.1. Let $O$ be an orbisurface with $s$ cone points of orders $m_{1}, \ldots, m_{s}$. Then we define the (orbifold) Euler characteristic of $O$ to be

$$
\chi(O)=\chi\left(X_{O}\right)-\sum_{j=1}^{s}\left(1-\frac{1}{m_{j}}\right),
$$

where $\chi\left(X_{O}\right)$ is the Euler characteristic of the underlying topological space of $O$.

A Riemannian orbisurface is an orbisurface which is endowed with a Riemannian metric. The Gauss-Bonnet theorem gives the usual relationship between topology and geometry for these objects:

Theorem 3.2. Let $O$ be a Riemannian orbisurface. Then

$$
\int_{O} K d A=2 \pi \chi(O)
$$

where $K$ is the curvature and $\chi(O)$ is the orbifold Euler characteristic of $O$.

Since the volume of a Riemannian orbisurface is spectrally determined via Weyl's asymptotic formula (see [4]), we see that for a Riemannian orbisurface with given curvature, the spectrum determines the orbifold Euler characteristic. However, since the orbifold Euler characteristic involves both the genus of the underlying surface and the orders of the cone points in the orbisurface, it is not immediately clear that the spectrum determines the genus. In the case of compact orientable hyperbolic orbisurfaces, Theorem 1.1 says that the spectrum determines the orders of the cone points, and thus the genus. This observation proves

Proposition 3.3. Isospectral compact orientable hyperbolic orbisurfaces have the same underlying topological space.

\section{REFERENCES}

1. Emily B. Dryden. Geometric and Spectral Properties of Compact Riemann Orbisurfaces. Ph.D. Dissertation, Dartmouth College, Department of Mathematics, May 2004. http://www.math.mcgill.ca/ ${ }^{\sim}$ dryden/thesis.pdf

2. E. Dryden, C. Gordon, S. Greenwald, D. Webb and S. Zhu. Asymptotic Expansion of the Heat Kernel for Orbifolds. Preprint, 2006.

3. J. Elstrodt, F. Grunewald, and J. Mennicke. Groups acting on hyperbolic space. Springer Monographs in Mathematics. Springer-Verlag, Berlin, 1998. Harmonic analysis and number theory.

4. Carla Farsi. Orbifold spectral theory. Rocky Mountain J. Math., 31(1):215-235, 2001.

5. L. Guillopé and M. Zworski. The Wave Trace for Riemann Surfaces. GAFA, 9:1156-1168, 1999.

6. Dennis A. Hejhal. The Selberg trace formula for $\operatorname{PSL}(2, R)$. Vol. I. Springer-Verlag, Berlin, 1976. Lecture Notes in Mathematics, Vol. 548. 
7. Henryk Iwaniec. Spectral Methods of Automorphic Forms. American Mathematical Society, 2002. Graduate Studies in Mathematics, Vol. 53.

8. L. B. Parnovskiı. The Selberg trace formula and the Selberg zeta function for cocompact discrete subgroups $\mathrm{SO}_{+}(1, n)$. Funktsional. Anal. i Prilozhen., 26(3): 55-64, 1992.

9. Naveed Shams, Elizabeth Stanhope, and David L. Webb. One Cannot Hear Orbifold Isotropy Type. Preprint, 2005.

10. Elizabeth Stanhope. Spectral Bounds on Orbifold Isotropy. Annals of Global Analysis and Geometry, 27(4): 355-375, 2005.

11. William P. Thurston. The Geometry and Topology of Three-Manifolds, Electronic edition of 1980 lecture notes distributed by Princeton University, available at http://www.msri.org/publications/books/gt3m/ 